\title{
Metabolomics of prematurity: analysis of patterns of amino acids, enzymes, and endocrine markers by categories of gestational age
}

\author{
Kumanan Wilson ${ }^{1-3}$, Steven Hawken ${ }^{2-4}$, Robin Ducharme ${ }^{2-4}$, Beth K. Potter ${ }^{2-4}$, Julian Little ${ }^{4}$, Bernard Thébaud ${ }^{5-7}$ \\ and Pranesh Chakraborty 5,8
}

BACKGROUND: Prematurity may influence the levels of amino acids, enzymes, and endocrine markers obtained through newborn screening. Identifying which analytes are the most affected by degree of prematurity could provide insight into how prematurity impacts metabolism.

METHODS: Analytes from blood spots assayed by Newborn Screening Ontario between March 2006 and April 2009 were used in this analysis. We examined the associations between the degree of prematurity and the levels of amino acids, enzymes, and endocrine markers in all newborns with and without adjustment for birth weight, feeding status, sample timing, transfusion, and sex.

RESULTS: Our analysis included the following cohorts: 373,819 children born at term (>36wk gestation), 26,483 near-term children (33-36 wk gestation), 4,354 very premature children (28-32 wk gestation), and 1,146 extremely premature children (<28wk gestation). Of the amino acids showing consistent trends across categories of prematurity, the levels of three amino acids (arginine, leucine, and valine) were at least 50\% different between the cohorts of extremely premature and term children. The levels of 17-hydroxyprogesterone increased with increasing prematurity, while thyrotropin-stimulating hormone values consistently decreased with increasing prematurity. None of the three enzyme markers we examined showed a trend in levels across categories of prematurity.

CONCLUSION: This study demonstrates that children at different stages of prematurity are metabolically distinct. Future research should focus on the mechanism by which specific analytes are influenced by prematurity.

n population-wide newborn screening, dried blood spot samples are obtained usually within $24-72 \mathrm{~h}$ of birth from which levels of various analytes are measured $(1,2)$. In Ontario and several other jurisdictions, these analytes include markers of amino acid metabolism, fatty acid oxidation, and endocrine function (3-7). Analyte results are used to calculate risk for rare inborn errors of metabolism and endocrine disorders.
Children having positive screening results are sent for confirmatory testing.

It is generally recognized that, aside from the presence of a rare metabolic or endocrine disease, several other factors can affect the analytes measured for newborn screening. One of these is gestational age (GA) at birth (8-11). Prematurity may influence analyte levels through a variety of mechanisms, including increased catabolic stress, lessened maturation of metabolic pathways and/ or organ systems, and differential distribution of other confounding variables such as feeding status $(9,12)$. Identifying which analytes are the most affected by extent of prematurity could provide insight into how prematurity influences metabolism.

In Ontario, Canada, virtually every child undergoes newborn screening (13). Results for all analytes measured are stored along with key demographic variables. We examined the associations between different degrees of prematurity and subclinical levels of specific analytes, after removing screen positives/extreme values. In particular, we examined the associations between the degree of prematurity and the levels of amino acids, enzymes, and endocrine markers in all newborns with and without adjustment for sex, birth weight, supplemental feeding, timing of sample collection, and receipt of blood or blood product.

\section{RESULTS}

\section{Characteristics of Population}

Newborn screening analyte data for a total of 412,494 children born in Ontario between April 2006 and March 2009 were available at the Institute for Clinical Evaluative Sciences (Ottawa, Ontario, Canada). Of these, 405,802 also had data on GA and were therefore included in our analysis. Our analysis included 373,819 children born at term, 26,483 near-term children, 4,354 very premature children, and 1,146 extremely premature children. Table 1 presents the sex proportions, mean birth weights, median time of sample collection, percent receiving transfusion, and mode of infant feeding by category of prematurity. In our overall sample, $51.3 \%$ of infants were male and $48.7 \%$ were female with similar proportions in all 


\section{Articles | Wilson et al.}

Table 1. Demographics of study population

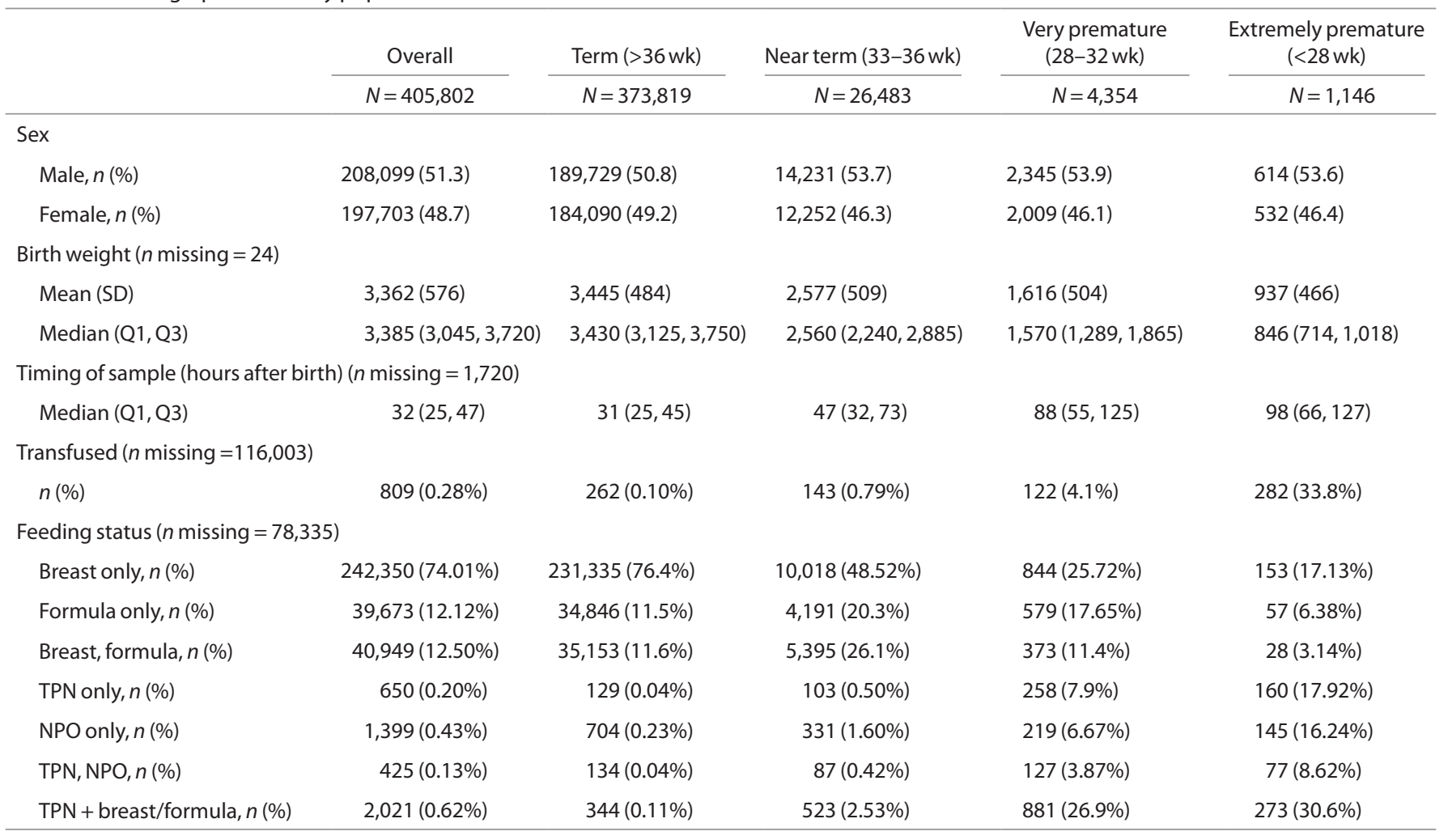

$\mathrm{NPO}$, nil per os; $\mathrm{Q} 1$, first quartile; $\mathrm{Q} 3$, third quartile; TPN, total parenteral nutrition.

categories of prematurity. The overall mean birth weight was $3,362 \mathrm{~g} ; 3,445 \mathrm{~g}$ in term infants, $2,577 \mathrm{~g}$ in near-term infants, $1,616 \mathrm{~g}$ in very premature infants, and $937 \mathrm{~g}$ in extremely premature infants. Timing of sample collection progressively increased with increasing prematurity (a median of $31 \mathrm{~h}$ in term infants and $98 \mathrm{~h}$ in extremely premature infants). Percent transfused also progressively increased with $0.1 \%$ of term children and $33.8 \%$ of extremely premature children being transfused.

\section{Unadjusted Analysis of Mean Analyte Levels by Degree of Prematurity}

Table 2 presents the unadjusted mean analyte levels in each category of prematurity, in addition to the differences between categories of prematurity expressed in units of SDs (SD in term children) and as percent differences from mean levels in term children.

Amino acids. We observed that 6 of the 10 amino acids studied (arginine, glycine, leucine, ornithine, phenylalanine, and valine) showed a consistent trend across categories of prematurity. Five of these amino acids (arginine, leucine, ornithine, phenylalanine, and valine) demonstrated rising values with increasing prematurity, whereas the level of glycine declined with increasing prematurity. Of the amino acids showing consistent trends, the largest difference was observed in the level of valine, with a difference of 2.24 SDs between extremely premature $($ mean $=176.4 \mu \mathrm{mol} / \mathrm{l})$ and term children
$($ mean $=108.0 \mu \mathrm{mol} / \mathrm{l})$, an increase of $63 \%$ in extremely premature as compared with term children.

Enzymes. None of the three enzyme markers we examined showed a trend in levels across categories of prematurity in the crude means comparisons (Table 2).

Endocrine markers. Both endocrine markers (thyrotropinstimulating hormone (TSH) and 17-hydroxyprogesterone (17OHP)) showed a consistent trend in values across categories of prematurity. The levels of 17-OHP increased with increasing prematurity, while the levels of TSH consistently decreased with increasing prematurity. The levels of 17-OHP in extremely premature children were 3.64 SDs (184\%) higher as compared with those in term children.

\section{Adjusted Analyses of Mean Analyte Levels by Degree of Prematurity}

After controlling for sex, birth weight, supplementation, timing of sample collection, and receipt of transfusion, we continued to observe the same linear trends across categories of GA in arginine, glycine, leucine, TSH, and 17-OHP (Table 3). In the adjusted model, a modest downward trend was also observed in alanine and methionine levels over increasing categories of prematurity. The largest differences between extremely premature and term infants were observed in the mean levels of leucine, arginine, TSH, and 17-OHP after adjustment for covariates. 
Table 2. Mean analyte values by prematurity status

\begin{tabular}{|c|c|c|c|c|c|c|c|}
\hline \multirow[b]{2}{*}{ Variable } & \multirow{2}{*}{$\begin{array}{c}\text { Term } \\
\text { Mean }(\mathrm{SD}) \\
(\mu \mathrm{mol} / \mathrm{l})\end{array}$} & \multicolumn{2}{|c|}{ Near term } & \multicolumn{2}{|c|}{ Very premature } & \multicolumn{2}{|c|}{ Extremely premature } \\
\hline & & $\begin{array}{l}\text { Mean (SD) } \\
(\mu \mathrm{mol} / \mathrm{l})\end{array}$ & $\begin{array}{l}\text { Pct diff; } \\
\text { (Std diffa) }\end{array}$ & $\begin{array}{l}\text { Mean (SD) } \\
(\mu \mathrm{mol} / \mathrm{l})\end{array}$ & $\begin{array}{l}\text { Pct diff; } \\
\text { (Std diffa) }\end{array}$ & $\begin{array}{c}\text { Mean (SD) } \\
(\mu \mathrm{mol} / \mathrm{l})\end{array}$ & $\begin{array}{l}\text { Pct diff; } \\
\text { (Std diffa) }\end{array}$ \\
\hline ALA & $274.3(82.6)$ & $258.6^{*}(86.1)$ & $-5.7 \% ;(-0.19)$ & $272.1(88.9)$ & $-0.80 \% ;(-0.03)$ & $250.3^{*}(83.1)$ & $-8.8 \% ;(-0.29)$ \\
\hline $\mathrm{ARG}^{\mathrm{b}}$ & $13.0(6.8)$ & $13.8^{*}(8.1)$ & $6.1 \% ;(0.12)$ & $20.4^{*}(12.5)$ & $56.8 \% ;(1.1)$ & $24.1 *(12.0)$ & $85.1 \% ;(1.63)$ \\
\hline LEU $^{\mathrm{b}}$ & $93.7(25.1)$ & $101.6^{*}(29.1)$ & $8.4 \% ;(0.32)$ & $128.9 *(38.9)$ & $37.5 \% ;(1.40)$ & $140.6^{*}(33.8)$ & $50.0 \% ;(1.87)$ \\
\hline MET & $24.8(6.6)$ & $25.6^{*}(7.6)$ & $3.2 \% ;(0.12)$ & $27.2^{*}(9.0)$ & $9.4 \% ;(0.36)$ & $26.6^{*}(8.7)$ & $7.1 \% ;(0.27)$ \\
\hline $\mathrm{ORN}^{\mathrm{b}}$ & $110.1(38.4)$ & $110.3(45.4)$ & $0.16 \% ;(0.01)$ & $130.0 *(52.8)$ & $18.1 \% ;(0.52)$ & $139.0 *(46.3)$ & $26.2 \% ;(0.75)$ \\
\hline $\mathrm{PHE}^{\mathrm{b}}$ & $58.7(12.2)$ & $59.7^{*}(13.1)$ & $1.6 \% ;(0.08)$ & $67.1 *(16.5)$ & $14.3 \% ;(0.69)$ & $70.5^{*}(15.2)$ & $20.1 \% ;(0.97)$ \\
\hline GALT & $253.5(84.1)$ & $240.0^{*}(82.7)$ & $-5.3 \% ;(-0.16)$ & $241.3^{*}(82.5)$ & $-4.8 \% ;(-0.15)$ & $190.8^{*}(71.7)$ & $-24.7 \% ;(-0.75)$ \\
\hline $\mathrm{BIO}$ & $43.5(11.9)$ & $41.7^{*}(11.7)$ & $-4.1 \% ;(-0.15)$ & $42.8(12.1)$ & $-1.6 \% ;(-0.06)$ & $38.8^{*}(11.1)$ & $-10.8 \% ;(-0.39)$ \\
\hline IRT & $24.4(12.9)$ & $25.2^{*}(11.9)$ & $3.1 \% ;(0.06)$ & $24.4(11.8)$ & $-0.33 \% ;(-0.01)$ & $28.4^{*}(15.6)$ & $16.2 \% ;(0.31)$ \\
\hline \multicolumn{8}{|c|}{ Endocrine markers } \\
\hline $\mathrm{TSH}^{\mathrm{b}}$ & $3.8(2.2)$ & $3.1 *(2.2)$ & $-18.7 \% ;(-0.31)$ & $1.9 *(1.6)$ & $-50.1 \% ;(-0.84)$ & $1.54^{*}(1.44)$ & $-58.9 \% ;(-0.99)$ \\
\hline 17-OHPb & $19.7(9.9)$ & $29.8^{*}(18.2)$ & $51.0 \% ;(1.01)$ & $40.4^{*}(24.1)$ & $105.0 \% ;(2.08)$ & $55.9 *(25.8)$ & $183.7 \% ;(3.64)$ \\
\hline \multicolumn{8}{|c|}{$\begin{array}{l}\text { 17-OHP, 17-hydroxyprogesterone; ALA, alanine; ARG, arginine; BIO, biotinidase; CIT, citruline; GALT, galactose } 1 \text { phosphate uridyl transferase; GLY, glycine; IRT, immunoreactive } \\
\text { trypsinogen; LEU, leucine; MET, methionine; ORN, ornithine; PCt diff, percent difference; PHE, phenylalanine; Std diff, standardized difference; TSH, thyrotropin-stimulating hormone; } \\
\text { TYR, tyrosine;VAL, valine. }\end{array}$} \\
\hline $\begin{array}{l}\text { aStandardiz } \\
\text { from term }\end{array}$ & $\begin{array}{l}\text { d difference }=(\mathrm{me} \\
\text { hildren } P<0.001 \text {. }\end{array}$ & -mean 2)/SD (calcu & for term children only & alyte demonstrate & insistent trend across & gories of prematurit) & gnificantly differe \\
\hline
\end{tabular}

\section{Restricted "Base Case" Analysis}

In our restricted analysis, we continued to observe the same linear trends across categories of GA in arginine, glycine, phenylalanine, TSH, and 17-OHP, as well as the same modest downward trend observed for alanine in the adjusted model (Table 4). In the restricted analysis, upward linear trends were also observed in methionine and immunoreactive trypsinogen. The largest differences between extremely premature and term infants were observed for arginine and 17-OHP.

\section{Heat Map Analysis}

Figure 1 presents the correlational heat map for all children. Darker red areas indicate stronger positive correlations between analytes, and darker blue areas indicate stronger negative correlations between analytes. The overall correlational heat map shows that the vast majority of correlations between pairs of analytes were positive; i.e., the higher the level of one analyte the higher the level of the other. Ten of the 120 correlations were negative; the negative correlations were generally weaker than the positive correlations. The strongest positive correlation was observed between valine and leucine ( $\rho=$ $0.77)$; variation in the value of one of these analytes explained $59 \%$ of the variation of the other. The strongest negative correlation was observed between leucine and TSH $(\rho=-0.249$;
$6.2 \%$ of variation of one of the analytes was predicted by the level of the other).

Figure 2 presents the difference heat map that highlights the pairs of analytes in which the correlations differed between term and premature infants. We observed the largest differences in correlations $(\Delta \rho)$ between term and premature children involving tyrosine (mean absolute difference in correlation between tyrosine and other analytes, $\Delta \rho=0.113)$, valine $(\Delta \rho=0.098)$, arginine $(\Delta \rho=0.093)$, and leucine $(\Delta \rho=0.092)$. The Supplementary Video S1 online demonstrates the changing correlations with progressive decrease in prematurity.

\section{DISCUSSION}

Our analysis confirms that infants born at different GAs are metabolically distinct, with consistent trends observed over increasing degrees of prematurity both in endocrine markers and in several amino acids measured in an unadjusted analysis. No trends were noted in the enzymes measured. Although differences in metabolic profile by degree of prematurity have been previously observed, this previous work has primarily focused on how GA may contribute to increased false-positive rates, with the goal of improving the precision of newborn screening in this population $(8,9,14)$. In addition to this important objective, we propose that examining the specific analytes whose levels are influenced by prematurity 


\section{Articles | Wilson et al.}

Table 3. Mean analyte values by prematurity status (after adjustment for birth weight, sex, feeding status, timing of sample collection, and transfusion status) ${ }^{\mathrm{a}}$

\begin{tabular}{|c|c|c|c|c|c|c|c|c|}
\hline \multirow[b]{2}{*}{ Variable } & \multicolumn{2}{|c|}{$\begin{array}{c}\text { Term } \\
(N=296,812)\end{array}$} & \multicolumn{2}{|c|}{$\begin{array}{l}\text { Near term } \\
(N=20,237)\end{array}$} & \multicolumn{2}{|c|}{$\begin{array}{l}\text { Very premature } \\
\qquad(N=3,234)\end{array}$} & \multicolumn{2}{|c|}{$\begin{array}{l}\text { Extremely premature } \\
\qquad(N=881)\end{array}$} \\
\hline & $S D^{b}$ & $\begin{array}{c}\text { Mean }(\mu \mathrm{mol} / \mathrm{l}) \\
(\mathrm{StdErr})\end{array}$ & $\begin{array}{c}\text { Mean }(\mu \mathrm{mol} / \mathrm{l}) \\
(\mathrm{Std} \text { Err })\end{array}$ & $\begin{array}{l}\text { Pct diff; } \\
\text { (Std diffe) }\end{array}$ & $\begin{array}{l}\text { Mean }(\mu \mathrm{mol} / \mathrm{l}) \\
\quad(\mathrm{Std} \text { Err })\end{array}$ & $\begin{array}{l}\text { Pct diff; } \\
\text { (Std diffe) }\end{array}$ & $\begin{array}{l}\text { Mean }(\mu \mathrm{mol} / \mathrm{l}) \\
\quad(\mathrm{Std} \text { Err })\end{array}$ & $\begin{array}{l}\text { Pct diff; } \\
\text { (Std diff') }\end{array}$ \\
\hline \multicolumn{9}{|c|}{ Amino acids } \\
\hline $\mathrm{ALA}^{\mathrm{d}}$ & 82.6 & $288.4(1.4)$ & $257.0 *(1.47)$ & $-10.9 \% ;(-0.38)$ & $247.6^{*}(1.70)$ & $-14.2 \% ;(-0.49)$ & $223.7^{*}(2.6)$ & $-22.4 \% ;(-0.78)$ \\
\hline$G L Y^{d}$ & 131.4 & $539.9(2.4)$ & $510.6^{*}(2.5)$ & $-5.4 \% ;(-0.22)$ & $492.3^{*}(3.14)$ & $-8.8 \% ;(-0.36)$ & $447.0^{*}(4.21)$ & $-17.2 \% ;(-0.71)$ \\
\hline LEU $^{d}$ & 25.1 & $102.6(0.74)$ & $105.6 *(0.69)$ & $2.9 \% ;(0.12)$ & $116.3^{*}(0.57)$ & $13.4 \% ;(0.55)$ & $126.6 *(0.89)$ & $23.4 \% ;(0.96)$ \\
\hline $\mathrm{MET}^{\mathrm{d}}$ & 6.6 & $29.6(0.12)$ & $29.0 *(0.13)$ & $-2.1 \% ;(-0.10)$ & $28.5^{*}(0.15)$ & $-3.8 \% ;(-0.17)$ & $26.3 *(0.21)$ & $-11.2 \% ;(-0.51)$ \\
\hline ORN & 38.4 & $123.6(0.98)$ & $118.0 *(1.00)$ & $-4.5 \% ;(-0.15)$ & $119.4^{*}(0.89)$ & $-3.4 \% ;(-0.11)$ & $122.3(1.24)$ & $-1.1 \% ;(-0.03)$ \\
\hline \multicolumn{9}{|c|}{ Enzymes/coenzymes } \\
\hline GALT & 84.1 & $237.6(1.43)$ & $234.7^{*}(1.45)$ & $-1.2 \% ;(-0.03)$ & $244.3^{*}(2.25)$ & $2.8 \% ;(0.08)$ & $200.9^{*}(2.8)$ & $-0.15 \% ;(-0.44)$ \\
\hline $\mathrm{BIO}$ & 11.9 & $38.9(0.22)$ & $38.3^{*}(0.24)$ & $-1.7 \% ;(-0.06)$ & $40.4^{*}(0.38)$ & $3.9 \% ;(0.13)$ & $40.8^{*}(0.51)$ & $4.9 \% ;(0.16)$ \\
\hline IRT & 12.9 & $27.1(0.43)$ & $26.9(0.43)$ & $-0.5 \% ;(-0.01)$ & $25.6(0.63)$ & $5.3 \% ;(-0.11)$ & $27.7(1.10)$ & $0.02 \% ;(0.05)$ \\
\hline \multicolumn{9}{|c|}{ Endocrine markers } \\
\hline $\mathrm{TSH}^{\mathrm{d}}$ & 2.23 & $4.21(0.039)$ & $3.52^{*}(0.041)$ & $-16.4 \% ;(-0.31)$ & $2.76^{*}(0.050)$ & $-34.4 \% ;(-0.65)$ & $2.02 *(0.071)$ & $-52.0 \% ;(-0.98)$ \\
\hline 17-OHPd & 9.94 & $23.47(0.39)$ & $32.84^{*}(0.35)$ & $39.9 \% ;(0.94)$ & $42.10 *(0.33)$ & $79.4 \% ;(1.87)$ & $55.04 *(1.14)$ & $134.5 \% ;(3.18)$ \\
\hline
\end{tabular}

could provide valuable insights into the metabolic pathways that may be most affected by the factors associated with prematurity, including neonatal stress, maturation of enzyme processes and organ systems, and the differential distribution of covariates.

Levels of several amino acids increased with higher degrees of prematurity. These findings were most evident in arginine and valine levels, which were more than 50\% higher in extremely premature infants compared with those in term infants; and leucine levels, which were 50\% higher. All but one (methionine) of the four essential amino acids we measured showed a consistent trend toward higher levels with categories of increasing prematurity. Consistent trends were evident in three of the five nonessential amino acids (arginine, ornithine, and glycine). The two branch-chained amino acids we measured (valine and leucine) had progressively increased levels with increasing prematurity. Glycine was the only amino acid for which the levels consistently decreased with increasing prematurity. The endogenous demands for glycine are high, and there may be an inability to sufficiently replace this amino acid in premature children, contributing to the declining levels with increasing prematurity (15).
The higher levels of amino acids in the blood spots of premature infants may represent markers of increased catabolism in these children. Supplementation may also play a role. Previous studies have observed a suppression of catabolism and an increase in amino acid levels with supplementation in premature children (16). Amino acid levels in the blood can also be influenced by the renal clearance rate. Older premature infants have been found to have a lower rate of absorption of amino acids by the renal tubules (17). Another potential explanation for elevated levels of amino acids in premature infants is delayed hepatic maturation.

Both endocrine markers were markedly associated with category of GA. 17-OHP, a marker of adrenal function, was inversely associated with GA category, with levels in extremely premature infants nearly twice those observed in term infants. These higher levels of 17-OHP are likely markers of increased stress among premature infants. Previous studies have identified that 17-OHP levels are correlated with neonatal stress and are elevated at birth and reduce subsequently $(18,19)$. In contrast, the levels of TSH decreased with increasing prematurity. The levels of TSH in extremely premature children were $\sim 60 \%$ lower than those in term children. These differences could reflect different stages of 
Table 4. Mean analyte values by prematurity status restricted analysis: not transfused, breastfed only, sample collected $73 \mathrm{~h}$ to $1 \mathrm{wk}$ after birth, non-SGA10 (adjusted for birth weight, sex, and timing of sample)

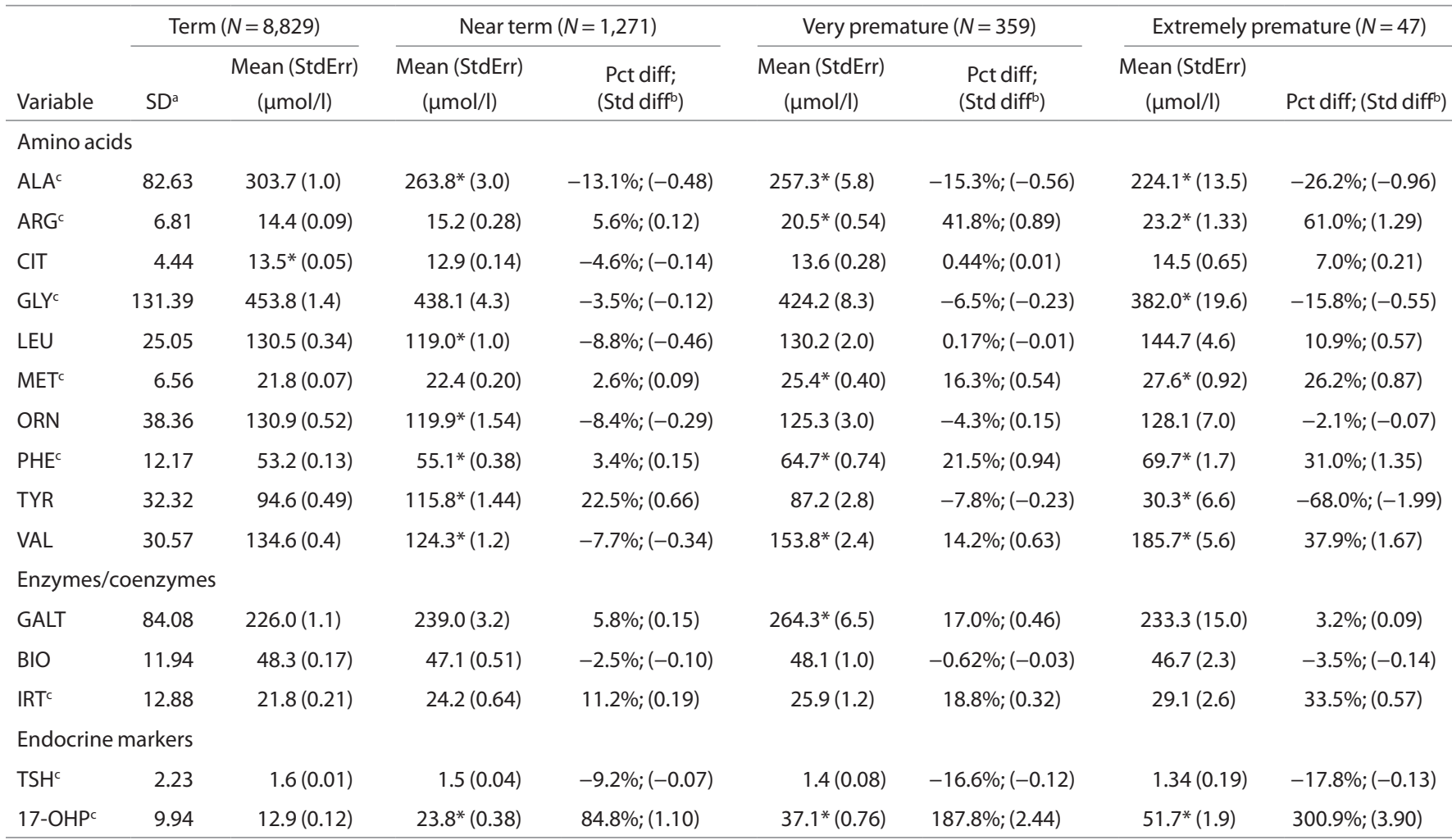

17-OHP, 17-hydroxyprogesterone; ALA, alanine; ARG, arginine; BIO, biotinidase; CIT, citruline; GALT, galactose 1 phosphate uridyl transferase; GLY, glycine; IRT, immunoreactive trypsinogen; LEU, leucine; MET, methionine; ORN, ornithine; Pct diff, percent difference; PHE, phenylalanine; SGA10, small for gestational age, below the 10th percentile for birth weight; StdErr, standard error; Std diff, standardized difference; TSH, thyrotropin-stimulating hormone; TYR, tyrosine; VAL, valine.

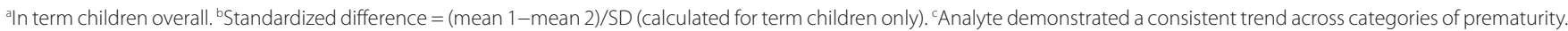
*Significantly different from term children $(P<0.001)$

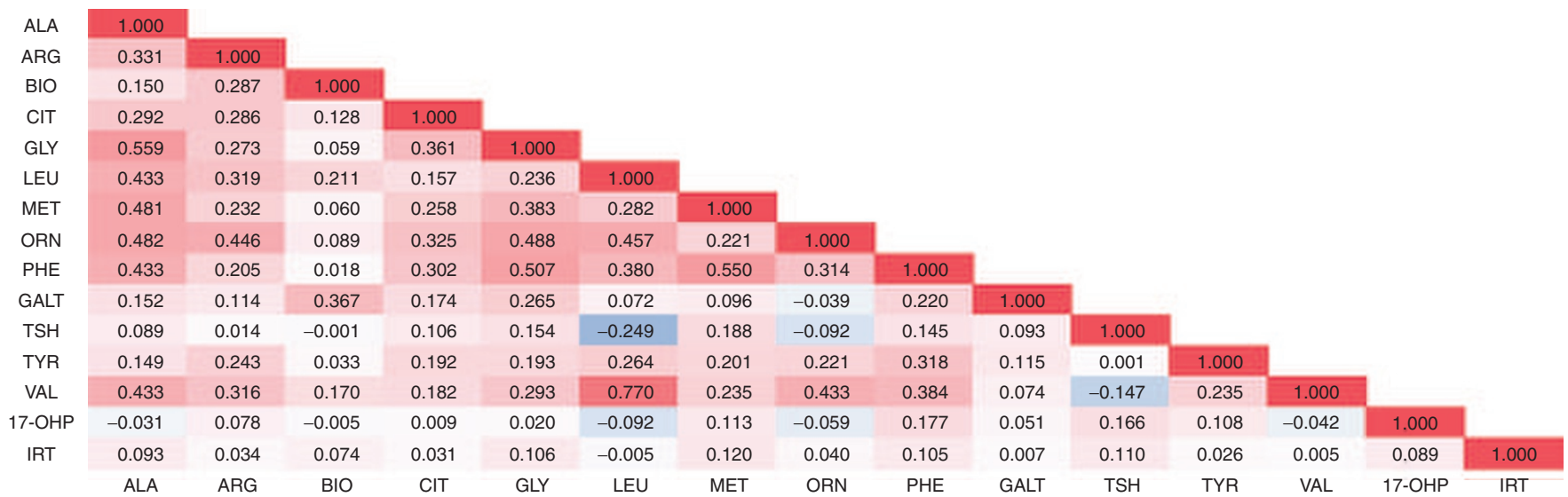

Figure 1. Correlational heat map for all children. Darker red areas indicate higher levels of positive correlation between analytes, and darker blue areas indicate higher levels of negative correlation between analytes. 17-OHP, 17-hydroxyprogesterone; ALA, alanine; ARG, arginine; BIO, biotinidase; CIT, citruline; GALT, galactose 1 phosphate uridyl transferase; GLY, glycine; IRT, immunoreactive trypsinogen; LEU, leucine; MET, methionine; ORN, ornithine; PHE, phenylalanine; TSH, thyrotropin-stimulating hormone; TYR, tyrosine; VAL, valine.

maturation of the pituitary and thyroid. Previous studies have also observed that premature children have lower levels of TSH and free $\mathrm{t} 3$ and $\mathrm{t} 4$ that progressively increase as levels of prematurity decrease $(20,21)$.

The levels of three enzymes we examined did not follow a clear pattern of effect with increasing prematurity. Given that enzymes have housekeeping functions, they would not be expected to vary linearly by the degree of prematurity, unlike amino acids and hormones.

The adjusted analysis and the restricted analysis altered the association between the level of prematurity and analyte level in several instances. The analytes that were consistently 


\begin{tabular}{|c|c|c|c|c|c|c|c|c|c|c|c|c|c|c|c|}
\hline ALA & 0.000 & & & & & & & & & & & & & & \\
\hline ARG & -0.037 & 0.000 & & & & & & & & & & & & & \\
\hline BIO & -0.049 & 0.006 & 0.000 & & & & & & & & & & & & \\
\hline CIT & -0.016 & -0.024 & -0.070 & 0.000 & & & & & & & & & & & \\
\hline GLY & 0.017 & 0.092 & 0.005 & 0.099 & 0.000 & & & & & & & & & & \\
\hline LEU & -0.075 & -0.243 & 0.003 & -0.130 & 0.062 & 0.000 & & & & & & & & & \\
\hline MET & -0.012 & -0.112 & -0.069 & -0.054 & -0.026 & -0.144 & 0.000 & & & & & & & & \\
\hline ORN & -0.043 & -0.093 & -0.057 & -0.016 & 0.081 & -0.115 & -0.096 & 0.000 & & & & & & & \\
\hline PHE & 0.037 & -0.118 & 0.006 & 0.052 & 0.062 & -0.124 & -0.009 & -0.032 & 0.000 & & & & & & \\
\hline GALT & -0.014 & 0.053 & 0.027 & 0.054 & 0.010 & 0.024 & -0.024 & 0.004 & 0.014 & 0.000 & & & & & \\
\hline TSH & 0.104 & 0.140 & 0.048 & 0.056 & -0.058 & 0.059 & 0.082 & 0.070 & 0.107 & 0.004 & 0.000 & & & & \\
\hline TYR & 0.034 & 0.208 & -0.086 & 0.063 & 0.091 & 0.188 & 0.088 & 0.202 & 0.236 & 0.042 & 0.011 & 0.000 & & & \\
\hline VAL & -0.002 & -0.232 & 0.050 & -0.050 & 0.113 & -0.056 & -0.146 & -0.104 & -0.113 & 0.064 & 0.097 & 0.275 & 0.000 & & \\
\hline 17-OHP & 0.082 & -0.005 & 0.061 & 0.106 & 0.111 & -0.127 & 0.100 & -0.024 & 0.009 & 0.076 & 0.207 & 0.161 & -0.135 & 0.000 & \\
\hline \multirow[t]{2}{*}{ IRT } & 0.038 & -0.026 & -0.009 & 0.008 & 0.021 & 0.031 & 0.022 & -0.015 & 0.039 & 0.030 & 0.032 & -0.014 & -0.029 & 0.048 & 0.000 \\
\hline & ALA & ARG & $\mathrm{BIO}$ & $\mathrm{CIT}$ & GLY & LEU & MET & ORN & PHE & GALT & TSH & TYR & VAL & 17-OHP & IRT \\
\hline Sum of Abs Diffs & 0.561 & 1.389 & -0.545 & 0.798 & 0.848 & 1.381 & 0.984 & 0.953 & 0.958 & 0.440 & 1.075 & 1.698 & 1.465 & 1.253 & 0.363 \\
\hline Avg of Abs Diffs & 0.037 & 0.093 & 0.036 & 0.053 & 0.057 & 0.092 & 0.066 & 0.064 & 0.064 & 0.029 & 0.072 & 0.113 & 0.098 & 0.084 & 0.024 \\
\hline
\end{tabular}

Figure 2. Heat map demonstrating differences in correlation between term and premature children. Darker red areas indicate higher levels of positive change in correlation between analytes, and darker blue areas indicate higher levels of negative change in correlation between analytes. 17-OHP, 17-hydroxyprogesterone; Abs Diffs, absolute differences; ALA, alanine; ARG, arginine; BIO, biotinidase; CIT, citruline; GALT, galactose 1 phosphate uridyl transferase; GLY, glycine; IRT, immunoreactive trypsinogen; LEU, leucine; MET, methionine; ORN, ornithine; PHE, phenylalanine; TSH, thyrotropinstimulating hormone; TYR, tyrosine; VAL, valine.

affected by GA in all three types of analyses were arginine, glycine, TSH, and 17-OHP. The differences between the results of the adjusted analysis and the restricted analysis could either represent the direct impact of the differential distribution of covariates, or the fact that the covariates are likely associated with poorer health status and may be indicators of catabolic stress. We hypothesize that analytes not impacted by adjustment for covariates may be those primarily affected by the impact of prematurity on the maturation of pathways rather than by general poor health or stress. Our restricted analysis attempted to control for this by fixing the covariates at levels expected in a term child; however, the analysis was limited by power issues in the extremely premature cohort.

The heat map analysis demonstrated correlations between analytes in all children and illustrated how these correlations changed with prematurity. Our use of difference heat maps to explore the latter is a novel methodology. We observed the largest differences in correlation among the four amino acids: tyrosine, valine, arginine, and leucine. Given that the metabolic processes involved in the production, utilization, and elimination of these analytes are dynamic, changes in correlations may provide clues as to how the relationship between analytes in these processes may be altered by prematurity.

A key strength of our study is that, to the best of our knowledge, this is one of the largest analyses conducted on this question. Furthermore, because newborn screening analytes are collected on over $99 \%$ of the Ontario population, our findings represent a census of the population and thus are not subject to selection bias. Limitations of the study relate to imprecision in the measurement of the analytes, especially those with lower concentrations. However, the impact of imprecision in the measurements in terms of random error is offset by our large sample size. A potential limitation of the study is the possible impact of unmeasured confounding on the relationship between category of prematurity and analyte levels. While we were able to explore the effects of factors such as sex, birth weight, timing of sample collection, receipt of transfusion, and feeding status, other potential confounders may have distorted the relationship between category of prematurity and analyte level $(8,14,22)$. Therefore, the relationships we observed in this study are best interpreted as associations between analytes and prematurity, or factors associated with prematurity such as catabolism, immaturity of enzyme systems, and organ development.

Our study has confirmed previously recognized associations between endocrine markers and GA at birth and identified novel relationships between GA at birth and levels of specific amino acids. Further research should focus on the impact of prematurity on the metabolic pathways involving the amino acids we identified as being associated with GA at birth. We also hypothesize that in term, normal-birth-weight infants, levels of these analytes could be indicators of infant vulnerability. Accordingly, future studies should examine the relationship between the analytes we identified and subsequent outcomes in term, normal-birth-weight infants. We also believe that metabolomic data collected at various stages of neonatal maturation in a complete population have the potential to make a large contribution to scientific understanding of the development of human metabolic pathways.

\section{METHODS}

This study was approved by the Ottawa Hospital Research Ethics Board. As this study was performed under strict conditions within the Institute for Clinical Evaluative Sciences, a Prescribed Entity under Ontario Canada's privacy legislation, express consent was not required for the use of anonymized health administrative data. Data on screening analytes from blood spots assayed by Newborn Screening Ontario between March 2006 and April 2009 were used in this analysis. We evaluated the levels of 15 specific analytes, including 10 amino acids, 3 enzymes, and 2 endocrine markers. The amino acids studied include arginine, phenylalanine, alanine, leucine, ornithine, citruline, tyrosine, glycine, methionine, and valine. Levels of amino acids were measured using tandem mass spectrometry. The enzymes we investigated were biotinidase, galactose 1 phosphate uridyl transferase, and immunoreactive trypsinogen. The two endocrine markers were TSH and 17-OHP, measured using immunoassays. For 
the majority of the analytes studied, values were available for children born in all $3 \mathrm{y}$ of study. Analysis of biotinidase, galactose 1 phosphate uridyl transferase, and 17-OHP by Newborn Screening Ontario began in 2007, and immunoreactive trypsinogen analysis began in 2008. In this study, we did not examine acylcarninitine levels, which will be analyzed in a separate study. Since the aim of our study was to look for trends in subclinical analyte levels, extremely low and high outliers for each analyte were removed. This was achieved by removing identified screen positives in addition to excluding observations below the 0.01th percentile and above the 99.99th percentile, effectively removing all screen positives from the analysis.

Using encrypted individual Ontario Health Insurance Plan numbers, screening data were linked to patient records in Ontario's health administrative data housed at the Institute for Clinical Evaluative Sciences, a repository of linked health information databases. We conducted our analysis on screened children for whom data were available on GA.

Based on GA at birth, we classified infants into four categories of prematurity: term ( $>36 \mathrm{wk}$ gestation), near term $(33-36 \mathrm{wk}$ gestation), very premature (28-32 wk gestation), and extremely premature $(<28 \mathrm{wk}$ gestation). All study data were housed at the Institute for Clinical Evaluative Sciences and were individually linked to the Ontario Registered Persons Database. The Registered Persons Database includes all Ontario residents who have ever been issued an Ontario Health Insurance Program number (i.e., nearly all residents of the province).

We examined differences in levels of amino acids, enzymes, and endocrine markers by the extent of prematurity. We computed means for each analyte within each category of GA and compared the means between categories using: (i) standardized differences between term and premature children (differences were expressed in units of SD using the SD of the term category as the benchmark) and (ii) percent differences (differences were expressed as a percentage of the mean analyte level in term children).

To investigate the potential impact of covariates, we used linear models to test the robustness of relationships between prematurity categories and mean analyte levels after controlling for sex, birth weight, mode of feeding, timing of sample collection, and receipt of blood or blood product transfusion. For feeding and transfusion status, we used data as recorded on the newborn screening record (i.e., feeding status proximal to the blood spot collection). Possible values included breast, formula, total parenteral nutrition, nil per os, and combinations thereof.

We also conducted a restricted "base case" analysis in which the category or range of a number of important covariates were fixed at a level consistent with those of healthy infants. In this analysis, we compared children across the levels of prematurity who: (i) were not below the 10th percentile for birth weight given their GA (SGA10), (ii) were exclusively breastfed at the time of sample collection, (iii) did not receive any blood transfusion or other blood products, and (iv) had their blood spot sample collected between $72 \mathrm{~h}$ and $1 \mathrm{wk}$ of age. Our choice of window for sample collection took the distribution of observed values into consideration by excluding extreme values without eliminating the majority of extremely premature infants. We conducted this restricted analysis because trends in covariates with increasing prematurity may also be associated with increased catabolic stress, making it difficult to separate potentially causal effects. Furthermore, some of these important covariates may be effect modifiers and may significantly interact with the relationship between analyte levels and prematurity. $P$ values were calculated for two-tailed tests of differences between least-squares means for each prematurity group vs. term children.

To examine the interrelated nature of the analytes as markers of common metabolic pathways that may be influenced by GA, we generated Pearson correlation heat maps among pairs of analytes in all infants, term infants and premature infants. In addition, we generated a difference heat map to highlight the pairs of analytes in which the Pearson correlations were different between term and preterm children.

\section{SUPPLEMENTARY MATERIAL}

Supplementary material is linked to the online version of the paper at http:// www.nature.com/pr

\section{STATEMENT OF FINANCIAL SUPPORT}

This study was supported by the Institute for Clinical Evaluative Sciences, which is funded by an annual grant from the Ontario Ministry of Health and Long-Term Care. J.L. is supported by the Canada Research Chair program. K.W. is supported by the Chair in Public Health Policy at The Ottawa Hospital, the University of Ottawa's Department of Medicine, and the Ottawa Hospital Research Institute.

Disclaimer: The opinions, results, and conclusions reported in this article are those of the authors and are independent from the funding sources. No endorsement by the Institute for Clinical Evaluative Sciences or the Ontario MOHLTC is intended or should be inferred.

\section{REFERENCES}

1. Wilson JM, Jungner YG. [Principles and practice of mass screening for disease]. Bol Oficina Sanit Panam 1968;65:281-393.

2. Newborn Screening Ontario. Information for Parents. (http://www.newbornscreening.on.ca/bins/content_page.asp?cid=6-12\&lang=1.) Accessed 3 June 2013

3. Therrell BL, Adams J. Newborn screening in North America. J Inherit Metab Dis 2007;30:447-65.

4. Bodamer OA, Hoffmann GF, Lindner M. Expanded newborn screening in Europe 2007. J Inherit Metab Dis 2007;30:439-44.

5. Borrajo GJ. Newborn screening in Latin America at the beginning of the 21st century. J Inherit Metab Dis 2007;30:466-81.

6. Padilla CD, Therrell BL. Newborn screening in the Asia Pacific region. J Inherit Metab Dis 2007;30:490-506.

7. Saadallah AA, Rashed MS. Newborn screening: experiences in the Middle East and North Africa. J Inherit Metab Dis 2007;30:482-9.

8. Oladipo OO, Weindel AL, Saunders AN, Dietzen DJ. Impact of premature birth and critical illness on neonatal range of plasma amino acid concentrations determined by LC-MS/MS. Mol Genet Metab 2011;104:476-9.

9. Slaughter JL, Meinzen-Derr J, Rose SR, et al. The effects of gestational age and birth weight on false-positive newborn-screening rates. Pediatrics 2010;126:910-6.

10. Dhondt JL. [Prematurity and neonatal screening]. Arch Pediatr 2008;15:Suppl 1:S7-S11.

11. Zaffanello M, Maffeis C, Zamboni G. Multiple positive results during a neonatal screening program: a retrospective analysis of incidence, clinical implications and outcomes. J Perinat Med 2005;33:246-51.

12. Kaye CI, Accurso F, La Franchi S, et al.; Committee on Genetics. Introduction to the newborn screening fact sheets. Pediatrics 2006;118:1304-12.

13. Wilson K, Kennedy SJ, Potter B, Geraghty MT, Chakraborty P. Developing a national newborn screening strategy for Canada. Health Law Rev 2010;18:21-9.

14. Ryckman KK, Berberich SL, Shchelochkov OA, Cook DE, Murray JC Clinical and environmental influences on metabolic biomarkers collected for newborn screening. Clin Biochem 2013;46:133-8.

15. Salway J. Biosynthesis of the non-essential amino acids. In: Metabolism at a Glance, 3rd edn. Oxford, United Kingdom: Blackwell Publishing, 2004:78-9.

16. te Braake FW, van den Akker CH, Wattimena DJ, Huijmans JG, van Goudoever JB. Amino acid administration to premature infants directly after birth. J Pediatr 2005;147:457-61.

17. Sereni F, McNamara H, Shibuya M, Kretchmer N, Barnett HL. Concentration in plasma and rate of urinary excretion of amino acids in premature infants. Pediatrics 1955;15:575-85.

18. Ersch J, Beinder E, Stallmach T, Bucher HU, Torresani T. 17-Hydroxyprogesterone in premature infants as a marker of intrauterine stress. J Perinat Med 2008;36:157-60.

19. Linder N, Davidovitch N, Kogan A, et al. Longitudinal measurements of 17alpha-hydroxyprogesterone in premature infants during the first three months of life. Arch Dis Child Fetal Neonatal Ed 1999;81:F175-8.

20. Thorpe-Beeston JG, Nicolaides KH, Felton CV, Butler J, McGregor AM. Maturation of the secretion of thyroid hormone and thyroid-stimulating hormone in the fetus. N Engl J Med 1991;324:532-6.

21. Fisher DA. The hypothyroxinemia [corrected] of prematurity. J Clin Endocrinol Metab 1997;82:1701-3.

22. Balk KG. Recommended newborn screening policy change for the NICU infant. Policy Polit Nurs Pract 2007;8:210-9. 